ACTA UNIVERSITATIS LODZIENSIS

Folia Litteraria Romanica 15, 2020

https://doi.org/10.18778/1505-9065.15.09

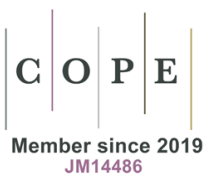

\author{
Loïc Capron \\ (iD) ORCID ID : 0000-0003-4029-4894 \\ loicapron@gmail.com
}

\title{
Un miroir du mépris : \\ Guy Patin contre Théophraste Renaudot (1638-1648)
}

\begin{abstract}
RÉSUMÉ
De 1638 à 1648, tout a opposé les deux médecins parisiens Théophraste Renaudot (1586-1653) et Guy Patin (1601-1672) : empirisme contre dogmatisme, paracelsisme contre galénisme, arrivisme politique contre mépris de la cour royale, Université de Montpellier contre Faculté de médecine de Paris... Sans doute attisé par leur ancienne camaraderie, leur duel a produit un foisonnement de libelles, auxquels s'ajoutèrent procès et pieds de nez dans un déchaînement réciproque de haine et de dédain. En 1643, après la mort de Richelieu et de Louis XIII, Patin pouvait impunément injurier son ennemi désarmé ; en disgrâce à la cour, Renaudot dut s'avouer vaincu. À la fin du XIX ${ }^{\mathrm{e}}$ siècle s'engagea une joute posthume entre les deux ennemis : Patin pour ses Lettres caustiques (rééditées en 1846) et Renaudot pour son invention du journalisme en France (La Gazette créée en 1631). L'opinion plaça le journaliste sur un piédestal et le fit jouir d'une immense célébrité en attachant son nom à celui d'un prix littéraire (1926).
\end{abstract}

MOTS-CLÉS - Bureau d'adresse, consultations charitables, Faculté de médecine de Paris, Gazette de France, Prix Théophraste Renaudot

“A Mirror of Disdain: Guy Patin v. Théophraste Renaudot (1638-1648)"

\section{SUMMARY}

Between 1638 and 1648, the two Parisian physicians Théophraste Renaudot (1586-1653) and Guy Patin (1601-1672) were rival in every matter: empiricism v. dogmatism, paracelsim v. galenism, political pushfulness v. contempt for courtism, Montpellier's University v. Paris's medical Faculty... Doubtless stirred up by their past companionship, their duel provoked a profusion of lampoons, followed by lawsuits and snubs, in a mutual explosion of hate and contempt. In 1643, Richelieu and Louis XIII being dead, Patin could abuse his disarmed enemy with complete impunity; in disgrace at court, Renaudot had to admit his defeat. At the end of the nineteenth century began a posthumous battle between the two enemies: Patin for his caustic Lettres (republished in 1846), and Renaudot for having been the inventor of French journalism ( $L a$ Gazette created in 1631). Public opinion set the journalist on a pedestal and gave him access to huge fame by giving his name to a literary award (1926). 
KEYWORDS - Bureau d'adresse (Employment agency), charity consultations, Paris's medical Faculty, Gazette de France, Prix Théophraste Renaudot (litterary award)

\title{
1. Le mépris français au XVII ${ }^{\mathrm{e}}$ siècle
}

\begin{abstract}
Mespris : rebut, desdain, tesmoignage du peu d'estime qu'on fait d'une chose. La chose la plus dure à supporter pour un honneste homme, c'est le mespris. Les braves font un généreux mespris de la vie. Un vray chrestien a du mespris pour toutes les vanitez mondaines. Un Prince fainéant tombe dans le mespris. Un avare est le rebut, le mespris du monde. On dit proverbialement, qu'il n'y a point de dette sitôt payée que le mespris ${ }^{1}$.
\end{abstract}

Les écrits polémiques ont surabondé durant la période dite baroque (1600-1661), dans les domaines politique, religieux ou scientifique. Le lecteur moderne reste souvent sidéré par la virulence et la cruauté, tant des attaques que des ripostes. Parmi une multitude de ces brûlots, rédigés en latin ou en français, l'édition de la Correspondance de Guy Patin ${ }^{2}$ m'a bien sûr mené aux furieux libelles qui ont accompagné sa longue querelle avec Théophraste Renaudot ; ces recherches me permettent d'ajouter quelques pièces à ce qui était déjà connu ${ }^{3}$.

\section{Protagonistes}

Théophraste Renaudot (Loudun 1586-Paris 1653) [fig. 1] était issu d'une famille calviniste aisée du Haut-Poitou. Il avait étudié la chirurgie à Paris (1602) puis été reçu docteur de l'Université de médecine de Montpellier (1606). Après avoir, semble-t-il, voyagé en Italie, il s'était installé à Loudun (1608) où il s'était marié et lié d'amitié avec l'évêque de Luçon, Armand Jean du Plessis de Richelieu (1585-1642), futur cardinal-ministre de Louis XIII, et avec François Leclerc du Tremblay (1577-1638), moine capucin plus connu sous le nom de père Joseph et d'Éminence grise du cardinal. En 1624, quand Richelieu entra au Conseil du roi, Renaudot jouissait déjà des titres de médecin ordinaire du roi autorisé à tenir des bureaux d'adresse ${ }^{4}$ et de commissaire général des pauvres du royaume. Fort de ses appuis et désireux d'en bien profiter, Renaudot s'était converti au catholicisme et installé à Paris en 1629-1630 pour donner libre cours à son talent d'inventeur, qui consistait souvent à promouvoir des idées qu'il avait empruntées à d'autres : établissement du Bureau

\footnotetext{
A. Furetière (1619-1688) : Dictionnaire universel, La Haye, A. et R. Leers, 1690, fo 3 Qqq 2 ro.

2 L. Capron, Correspondance complète et autres écrits de Guy Patin; URL : http://www.biusante. parisdescartes.fr/patin/; consulté le 09.01.2019.

3 G. Gilles de la Tourette, Théophraste Renaudot d'après des documents inédits, Paris, Plon, 1884.

4 Bureaux de placement et de petites annonces, dont Michel de Montaigne a attribué la première idée à son père (Essais, Liv. I, chap. 35).
} 
d'adresse et de rencontre dans l'île de la Cité (rue de la Calandre, Au grand Coq), bientôt assorti de consultations médicales charitables (gratuites), où n'œuvraient que des médecins étrangers ${ }^{5}$, de la fameuse Gazette (1631), puis de Conférences du Bureau d'adresse (1632) ${ }^{6}$ et enfin d'un mont-de-piété (1637).

Guy Patin (Hodencq-en-Bray 1601-Paris 1672) [fig. 2] était né dans une famille modeste de la campagne picarde (Beauvaisis). Il avait rompu avec sa famille pour venir étudier à Paris, en assurant sa propre subsistance. Ses bonnes dispositions lui avaient gagné le soutien de Jean Riolan, « le prince des anatomistes », qui devint son mentor et le resta jusqu'à sa mort (1657). Patin avait été reçu docteur régent de la Faculté de médecine de Paris en 1627. Ses talents littéraires et oratoires avaient bientôt fait de lui l'un des plus brillants espoirs du clan conservateur (dogmatique), alors majoritaire au sein de la Faculté (qui comptait quelque 120 docteurs).

Tous deux partageaient l'idéal de porter secours aux malades de toute condition sociale mais divergeaient dans leur manière de l'assouvir ; comme l'illustre leur attitude envers les panacées : Patin vouait aux gémonies ces extravagantes mixtures pharmaceutiques, accusant les empiriques et les charlatans qui les vendaient de tromper le peuple ; Renaudot avait donné le nom de polychreston à celle qu'il avait composée et dont il prônait les vertus ${ }^{7}$. Ni l'un ni l'autre n'accéda à la vaine gloire de soigner les grands du royaume : Patin n'y tenta même pas sa chance, se contentant d'une pratique issue de la noblesse de robe et de la bourgeoisie ; Renaudot intrigua fort au Palais-Cardinal, mais y demeura un homme de l'ombre, dont on protégeait les entreprises à condition qu'il rendît service avec sa Gazette et son utile réseau de correspondants. Tous deux étaient intelligents et écrivaient avec brio. Quant au savoir, enfin, celui de Patin était encyclopédique, mais stérile car engoncé dans les préjugés rétrogrades, là où celui de Renaudot était bien moins vaste, mais fécond car exploité sans grand scrupule de conscience.

5 La Faculté de médecine de Paris qualifiait d'étrangers les médecins qui n'étaient pas titulaires de ses grades (licence ou doctorat) permettant d'exercer dans la capitale. La seule exception tolérée concernait les médecins ordinaires de la cour (famille royale et princes du sang).

6 Premier périodique jamais imprimé en France, La Gazette de France était un hebdomadaire contenant des nouvelles politiques du monde entier, mais sous un jour toujours favorable au roi de France, à sa cour et à ses ministres. Ses numéros ordinaires paraissaient le samedi, avec un numéro extraordinaire mensuel. - Supplément scientifique puis journal indépendant de la Gazette, les Conférences $d u$ Bureau d'adresse publiaient, par centuries, les transactions de l'académie savante que Renaudot réunissait tous les lundis rue de la Calandre.

7 Description d'un médicament appelé polychreston, dispensé publiquement par Jacques Boisse, maître apothicaire en la ville de Loudun, le 4 décembre 1619. Avec la harangue faite sur ce sujet par Théophraste Renaudot, docteur en médecine, conseiller et médecin du roi, devant Messieurs les députés des Églises réformées de ce royaume, assemblés à Loudun par permission de sa Majesté. Dédié à Messieurs de l'Assemblée (Loudun, Quentin Maréchal, 1619, in-4º). Mêlant 83 extraits d'origine végétale ou animale, ce polychreston était un plagiat manifeste du polychreste que Jean Pidoux, médecin de Poitiers, avait décrit dans son livre intitulé Pestis cura et polychresti descriptio [Guérison de la peste et description du polychreste] (Poitiers, Jean Blancet, 1605, in- $8^{\circ}$ ). 


\section{Motifs de la querelle}

La Compagnie des médecins de Paris était installée rue de la Bûcherie, sur la rive gauche de la Seine, face à Notre-Dame. S'arrogeant l'exclusivité de la pratique et de l'enseignement médicaux dans la capitale, elle voyait prospérer les entreprises concurrentes de Renaudot, juste à côté d'elle, sur l'île de la Cité. Protégés par le roi Louis XIII (1601-1643) et par son principal ministre, Richelieu, ce médecin et ses adjoints, étrangers à l'Université de Paris, avaient l'outrecuidance d'y soigner gratuitement les pauvres, d'y prodiguer des conférences savantes et d'y préparer panacées douteuses et remèdes chimiques (antimoine, mercure, etc.), maintes fois proscrits par la Faculté. Qui plus est, Renaudot prostituait l'art médical en y mêlant de viles activités commerciales : Gazette, Bureau d'adresse, prêt sur gage.

Renaudot a ajouté à ces griefs corporatistes ses relations avec Patin, au temps où la correction d'épreuves d'imprimerie lui permettait de subsister :

Mais je me suis toujours ressouvenu de cette belle sentence que j'imprimais et que tu corrigeais au temps de notre première connaissance : que l'amitié doit sembler au lierre qui n'abandonne point, même après leur mort, les arbres qu'il a une fois embrassés. Ainsi, monsieur mon camarade que j'ai tant embrassé, surtout quand ce bon piot nous avait à tous deux ragaillardi les sens, je n'abandonnerai jamais la défense de ta réputation, même après l'avoir perdue, comme tu as fait par ces jolis petits livres que tu composes contre un homme avec qui tu ne gagneras rien $^{8}$.

Patin devait renier cette ancienne camaraderie, désormais gâtée par les ambitions réciproques, s'il voulait être crédible dans son rôle de vengeur masqué des docteurs régents parisiens contre Renaudot.

\section{Journal des principales hostilités}

\subsection{Mars 1638}

Deux fils de Théophraste Renaudot, Isaac et Eusèbe, respectivement nés en 1610 et 1613, avaient ensemble entamé leurs études à la Faculté de médecine de Paris en octobre 1634. Durant leur $4^{\mathrm{e}}$ année, comme voulait la règle, ils demandèrent à subir l'épreuve du baccalauréat (premier grade de médecine), pour laquelle la Compagnie avait élu quatre examinateurs le 30 janvier 1638, dont l'un était Patin. Ces docteurs

8 Réponse à l'Examen de la requête présentée à la reine par Me Théophraste Renaudot, portée à son auteur par Machurat, compagnon imprimeur (Paris, s.n., 1644, in- $4^{\circ}$ ). Renaudot y répondait anonymement à l'Examen de la Requête présentée à la reine par le gazetier (s.l., s.n., 4 novembre 1643, in- $4^{\circ}$ ), libelle anonyme que la Faculté de médecine de Paris avait fait écrire (sans doute par Patin) et imprimer contre son ennemi. — «Sembler» est à prendre pour « ressembler », et « piot » pour « vin ». 
régents, qui avaient pour première tâche de vérifier l'honorabilité et la capacité des candidats à devenir bacheliers, imposèrent aux deux postulants de prendre, le 21 mars, devant notaires, l'engagement suivant :

Par devant les notaires garde-notes du roi, notre Sire, au Châtelet de Paris, furent présents MM. Isaac et Eusèbe Renaudot, frères, maîtres ès arts en l'Université de Paris, étudiants en médecine, demeurant rue de la Calandre, au Grand Coq, paroisse [de] SaintGermain-le-Vieil ; lesquels ont promis à Messieurs de la Faculté de médecine de cette ville de Paris, qui l'ont ainsi requis d'eux, qu'au cas qu'ils aient l'honneur d'être reçus en la qualité de bacheliers de ladite Faculté et autres degrés d'icelle, comme ils l'espèrent et les supplient, ils n'exerceront point aucune des fonctions du Bureau d'adresse, mais s'adonneront entièrement à l'exercice de la médecine, à quoi ils se sont obligés sous l'hypothèque de tous et chacuns leurs biens'.

Toute honte bue, les deux Renaudot furent déclarés bacheliers le 27 mars suivant et parvinrent à être reçus licenciés en juin 1640, quand recommencèrent leurs ennuis académiques (voir infra).

\subsection{9 mars 1639}

En riposte à celle du Bureau d'adresse, la Faculté crée une consultation charitable : elle a lieu chaque samedi matin, rue de la Bûcherie.

\subsection{Septembre 1640 - juin 1641}

Les Lettres patentes du roi en faveur des pauvres, et particulièrement des malades, publiées le 25 septembre 1640, confirment les consultations charitables de Renaudot et l'exclusivité parisienne des remèdes qu'il y prescrit, « avec défenses à tous autres qu'à ceux qui auront pouvoir exprès de lui, de les imiter, altérer ou contrefaire »; elle concède au Bureau d'adresse le privilège de posséder des fourneaux pour fabriquer ses propres médicaments chimiques ${ }^{10}$.

Le 20 octobre, la Faculté attaque la légitimité des lettres devant le Parlement ; Renaudot gagne et attaque à son tour la Faculté, laquelle obtient du prévôt une interdiction que Renaudot exerce la médecine à Paris. Il imprime le Factum du

9 Commentaires de la Faculté de médecine de Paris, t. XIII (1636-1652), fo 48 ro ; Medic@; URL : http://www.biusante.parisdescartes.fr/histmed/medica/page?ms00001_00024x13\&p=97; consulté le 16.01.2019.

${ }^{10}$ Ces lettres patentes de Louis XIII sont imprimées à la fin des Consultations charitables pour les malades. Dédiées à Monseigneur de Noyers, secrétaire d'État (Paris, Bureau d'Adresse, 1640, in- $4^{\circ}$ ). L'installation de fourneaux métallurgiques était rigoureusement contrôlée par la Cour des monnaies car ils permettaient de fabriquer de fausses espèces métalliques. Les chimistes clandestins étaient souvent poursuivis comme faux-monnayeurs. 
procès d'entre Maître Théophraste Renaudot, docteur en médecine de la Faculté de Montpellier, médecin du roi, commissaire général des pauvres malades et valides de ce royaume, maître et intendant général des bureaux d'adresse de France, demandeur en requête présentée au Conseil privé du roi le 30 d'octobre 1640. Contre le doyen et les docteurs en médecine de la Faculté de Paris, défendeurs, sans que les qualités puissent préjudicier.

En décembre, la Faculté empêche les fils Renaudot de disputer leurs actes de doctorat ${ }^{11}$. Une première vague de libelles paraît : La Défense de la Faculté de médecine de Paris contre son calomniateur... ; Réponse de Théophraste Renaudot... au libelle fait contre les consultations charitables pour les pauvres malades $^{12}$; Avertissement à Théophraste Renaudot contenant les mémoires pour justifier les anciens droits et privilèges de la Faculté de médecine de Paris ; Remarques sur l'Avertissement à $M^{\mathrm{e}}$ Théophraste Renaudot, postées à son auteur par Maschurat, compagnon imprimeur. Richelieu, exaspéré, interdit la parution d'autres opuscules et exige que la Faculté permette aux fils Renaudot de devenir docteurs régents, ce qu'elle persiste à refuser.

\subsection{Nebulo et blatero}

En 1641, parut à Paris la première édition des œuvres complètes de Daniel Sennert ${ }^{13}$, préparée par Guy Patin. Le premier tome s'ouvre sur une épître dédicatoire, datée du $1^{\text {er }}$ juin, qu'il avait écrite en se cachant sous la signature des cinq libraires associés qui avaient imprimé le livre, et adressée à son collègue et ami René Moreau.

${ }^{11}$ Vespérie, doctorat proprement dit (avec remise du bonnet) et régence (acte pastillaire ou antéquodlibétaire) se disputaient successivement dans un délai de six semaines, après accord du doyen, soumis à l'avis de toute la Faculté.

${ }^{12}$ Entre bien d'autres écrits, ce livre (Paris, Bureau d'adresse, 1640, in- $4^{\circ}$ ) procure ce bel exemple du style de Renaudot et de sa manière d'argumenter contre Patin (p. 3) : " Que les esprits à qui j'ai à faire sont malaisés à contenter! Tandis que je fais servir de relâche [distraction] à mon emploi dans la médecine quelques autres exercices utiles au public, ils me blâment de ne m'adonner pas entièrement à cet art ; et lorsque je le veux exercer, même pour les pauvres, ils s'y opposent. Mon zèle me les rend ennemis. Ils ne me permettent pas de donner mon bien, mon temps et mon industrie sans procès. Ma charité, en un mot, leur est criminelle. Voyant ces mauvais effets d'une si bonne cause, si je me veux accorder à tout ce qui sera trouvé honnête aux deux parties, ils ne le veulent pas. Si je me défends et rends raison de mon droit à mes juges, un de ces esprits malades de la démangeaison d'écrire vomit sa bile sur du papier, en gardant néanmoins encore assez sur son visage olivâtre pour le faire appeler picrochole. Mais pource qu'il cèle son autre nom dans la foule de ses compagnons, j'ai trouvé à propos de ne le nommer pas en la consultation que je vais faire pour le guérir $»$.

${ }^{13}$ Danielis Sennerti D. Medici Opera omnia in tres tomos distincta [Euvres complètes de Daniel Sennert, docteur en médecine, réparties en trois tomes], Paris, par une Société de libraires, 1641, in-f $\mathrm{f}^{\mathrm{O}}$. Sennert (1572-1637) avait enseigné avec grand renom la médecine à l'Université de Wittemberg (Saxe). 


\section{Patin s'y attaque à Renaudot de manière à peine couverte quand il exhorte Moreau à rééditer ses œuvres :}

et devant tout le reste, cette brillante Défense, que vous avez publiée il y a peu de temps, pour la gloire et l'honneur de votre Faculté de médecine, contre un certain vaurien qui, sous un masque de feinte piété et de fourbe charité, entreprenait d'introduire en cette ville je ne sais quelles nouveautés et d'imposer partout les rêveries d'un esprit dérangé. Et si vous n'avez pas encore remporté une absolue victoire contre la férocité de cette hydre à brigander, tant par la force de votre éloquence que par le poids de vos raisonnements et de vos arguments, vous vous êtes uniquement préoccupé de l'honneur de la Faculté de médecine, que vous avez conservée saine et sauve contre les calomnies fort effrontées de ce babillard ; mais par cet exceptionnel bienfait, vous vous êtes aussi attaché toute la France car vous avez brisé l'orgueil d'une outrecuidance sans fond, vous avez entièrement émoussé les traits de celui qui, par ses fourberies, et contre toute loi humaine et divine, avait tendu le pire piège à ceux dont vous avez si remarquablement défendu l'innocence, la probité et l'honneur ${ }^{14}$ (trad. L.C.).

Cette double insulte, qui qualifiait Renaudot de nebulo [vaurien] et de blatero [babillard], bravait effrontément l'interdiction de Richelieu et attisa la querelle.

\subsection{2}

Après maints méandres, le 14 août 1642, devant le tribunal des Requêtes de l'Hôtel, eut enfin lieu le procès de Renaudot contre Patin pour l'épître du Sennert. Patin triompha, à l'immense joie de la Faculté, dont le doyen, Guillaume Du Val, consacra une page entière de ses Commentaires manuscrits à relater les faits, avec cet éloge de l'inculpé :

Mais voici que Patin se lève avec détermination et, plaidant lui-même sa cause avec éloquence, il réfute ses deux adversaires, il démontre et prouve que ces mots de vaurien et babillard ne sont pas injurieux ; et ce faisant, il convainc Renaudot d'erreur et le pique finement (car il avait le nez difforme, mangé et à demi rongé de vérole) par cette citation de saint Jérôme écrivant contre Bonasus [sic pour Onasus], son détracteur, orateur braillard qui avait un long nez (lettre 16, liv. I) : J'ai entrepris de couper un nez puant, que

${ }^{14}$ « Ac præ cæteris omnibus præclara illa, quam non ita pridem in uestræ facultatis Medicæ decus et honorem edidisti in lucem Apologia, aduersus quendam nebulonem, qui fictæ pietatis et charitatis non sinceræ laruam prætendens, in hanc Vrbem nouitates nescio quas inducere, et maleferiatæ mentis somnia passim obtrudere moliebatur. Neque uero dum de istius hydræ grassante jam iminanitate insignem uictoriam retulisti; tum facundiæ tuæ ui, tum rationum et argumentorum pondere, facultatis solum Medicæ honori consuluisti, quem ita sartum tectumque seruasti aduersus impudentissimas istius blateronis calumnias, uerumetiam singulari beneficio totam ipsam Galliam Tibi deuinxisti, dum elatos nimium tam perditæ frontis animos confregisti, illiusque tela plane retudisti, qui contra jus fasque omne technis suis pessime iis insidiabatur quorum innocentiam, probitatem et pietatem tam egregie defendisti ». 
le scrofuleux se tienne donc sur ses gardes. Par ce propos et bien d'autres, que $\mathrm{M}^{\mathrm{e}}$ Patin dit avec goût et distinction, en un discours improvisé mais érudit et de bonne longueur, les juges, admiratifs de son intelligence vive et acérée, et de ses arguments parfaitement placés et assurés, l'acquittèrent de bon cœur, en déclarant son accusation injuste. Les plaignants furent quant à eux déboutés, et interdits de toute action judiciaire et de tout procès, ce qu'on appelle en français hors de Cour et de procès, en présence de plusieurs milliers d'auditeurs ${ }^{15}$.

Dans les Avis au lecteur des premières éditions des lettres de Patin, VigneulMarville conte cette anecdote :

M. Patin gagna hautement sa cause contre lui [Renaudot], mais il consola sa partie en sortant de l'audience, l'ayant abordé d'un grand sang-froid : « Monsieur, lui dit-il, vous avez gagné en perdant. - Comment donc ? lui répondit Renaudot. - C'est, répliqua M. Patin, que vous étiez camus quand vous êtes entré au Palais, mais vous en sortez avec un pied de nez $»^{16}$.

Les événements se précipitèrent ensuite : 6 septembre, arrêt du Parlement ordonnant à la Faculté de recevoir les fils Renaudot docteurs ; 20 octobre, la Faculté accepte si la Cour interdit à leur père de continuer son activité médicale ; 2 décembre, Richelieu ayant accepté la condition, Isaac dispute le premier acte de son doctorat (vespérie) ; 4 décembre, mort de Richelieu, la Faculté interrompt les actes doctoraux des fils Renaudot qui ont perdu le plus puissant soutien de leur famille.

${ }^{15}$ Commentaires de la Faculté de médecine de Paris, t. XIII (1636-1652), $\mathrm{f}^{0} 141$ vo -142 r ${ }^{0}$; Medic@; URL: http://www.biusante.parisdescartes.fr/histmed/medica/page?ms00001_00024x13\&p=292; consulté le 16.01.2019 : « Sed ecce insurgit animose Patinus, et causam ipse suam diserte agens, utrumque acerrime confutat, Docet, probatque non esse uerba illa nebulo, blatero iniuriosa; atque interim arguit et acute perstringit Renaudotum (cui nasus est deformus erosus ac fere semesus ex uariolis) hac e Diuo Hieronymo, ad Bonasum Obtrectatorem et nasutum Rabulam scribente, ep. 16. 1. i selectarum sententia. Disposui nasum secare fotentem, timeat qui strumosus est. Quibus et aliis egregie et eleganter dictis atque extemporanea sed erudita et longiuscula oratione a Doctissimo Patino ennaratis, mirati iudices acre et promptum ipius ingenium rationesque appositissimas, et expeditissimas, eum facile absoluerunt tanquam inique accusatum; Accusantes autem emiserunt, omnibusque omni lite ac forensi Actione interdixerunt, quod uulgo dicitur Hors de Cour et de procez, præsentibus multis Auditorum millibus ».

${ }^{16}$ Lettres choisies de Feu Monsieur Guy Patin, docteur en médecine de la Faculté de Paris et professeur au Collège royal. Dans lesquelles sont contenues plusieurs particularités historiques, sur la vie et la mort des savants de ce siècle, sur leurs écrits et sur plusieurs autres choses curieuses, depuis l'an 1645 jusqu'en 1672, Francfort, pour J. L. Du-Four, 1683, in-12º pour la première des nombreuses éditions parues aux XVII et $\mathrm{XVIII}^{\mathrm{e}}$ siècles). - Comme on voit sur la figure 1 (et d'autres portraits ont encore accentué le trait), Renaudot avait le nez en pied de marmite, c'està-dire camus ou retroussé. Ses ennemis voyaient là un signe de syphilis tertiaire avec destruction des cartilages nasaux, mais d'autres diagnostics moins avilissants (dans les mentalités de l'époque) sont aujourd'hui envisageables. 


\subsection{3}

Encore protégé par le roi, Renaudot est autorisé à installer un hôtel de consultations charitables dans le faubourg Saint-Antoine ; mais Louis XIII meurt le 14 mai, laissant le gazetier sous la protection hésitante et fragile de la reine régente, Anne d'Autriche, et de Mazarin, bientôt appelé à devenir son principal ministre ${ }^{17}$.

Le 16 juillet, la Faculté dépose une requête devant le Conseil du roi contre l'extension des consultations charitables de Renaudot (et contre ses autres entreprises commerciales). Pour regagner quelque crédit à la cour, il publie la Requête présentée à la reine par Théophraste Renaudot en faveur des pauvres malades de ce royaume. Avec la participation très active de Patin, la Faculté fait réimprimer à ses frais le Factum que Renaudot avait publié en 1640, s'en sert pour justifier sa propre requête devant le prévôt de Paris contre les consultations charitables et leurs médecins étrangers, et fait paraître, le 4 novembre, l'Examen de la Requête présentée à la reine par le gazetier.

Le 9 décembre, le Châtelet donne raison à la Faculté contre Renaudot qui est condamné à ne plus exercer aucune de ses activités médicales parisiennes. Il fait aussitôt appel de cette sentence devant le Conseil du roi.

Le 17 décembre, Patin préside à la Faculté une thèse qu'il a lui-même écrite et qui a établi son renom dans toute l'Europe savante : Estne totus homo a natura morbus ? ${ }^{18}$ Parlant des ulcères qui rongent le visage, dans le $3^{\mathrm{e}}$ des 5 articles, il souille le nom de Renaudot avec cet infamant acrostiche :

J'ajouterai que les physiognomonistes veulent qu'un nez purulent imprègne l'esprit même de celui qui le porte, jusqu'à prendre pour axiome que la corruption des mœurs est la conséquence d'un nez pourri, ce qui est plausible ; en effet, à ce genre de nez qui parlent d'une voix aigrelette, c'est-à-dire odieusement et insupportablement, qu'on appelle partout des fripons, appartiennent les Ridicules, les Effrénés, les Néfastes, les Agités, les Vauriens, les Dissimulateurs, les Obscènes, les Trublions, les menteurs, les perfides, les envieux, les délateurs, les scandaleux, les infâmes,

17 Pour la reine, les choses semblent assez claires : mis à part Mazarin et quelques autres, elle détestait tous ceux qui avaient eu les faveurs de Richelieu, d'autant qu'en 1633 la Gazette avait laissé planer un doute sur des intelligences d'Anne d'Autriche avec l'Espagne; en outre, elle suivait pieusement les avis du Conseil de conscience et de son directeur, Vincent de Paul, le bras visible de la Compagnie du Saint-Sacrement qui tenait pour suspects tous ceux qui avaient un jour adhéré à la R.P.R. (Religion prétendue réformée), même après leur conversion au catholicisme. - Mazarin n'apprit que plus tard à se méfier des imprimés politiques, avec les mazarinades qui attisèrent la Fronde (1648-1653), mais il sut utiliser la Gazette qui servit toujours avec zèle les intérêts de la Couronne. Néanmoins, le cardinal ne semble pas avoir manifesté publiquement d'attachement pour Renaudot, mais ce point ferait un joli sujet pour des recherches approfondies.

18 «Par nature, l'homme n'est-il pas tout entier maladie?», sur les déterminants innées et acquis de la santé : j'ai transcrit, traduit et annoté cette thèse quodlibétaire dans mon édition des écrits de Guy Patin (http://www.biusante.parisdescartes.fr/patin/?do=pg\&let=8038; consulté le 18.01.2019). 
les injurieux, les criminels, ceux qu'aucune vertu ne rachète, malades de vices et forts de leur seule débauche, pétris de faux-semblant, et voués par nature à la fraude et à la calomnie ${ }^{19}$.

Renaudot connut très probablement cette nouvelle perfidie de Patin, mais préféra la mépriser pour la laisser tapie dans les lignes où son auteur l'avait enfouie, et d'où elle n'est d'ailleurs guère sortie depuis qu'elle y a été écrite.

\subsection{4-1648}

«Les degrés du Palais-Royal sont aussi glissants qu'aient jamais été ceux du Louvre » : la maxime de Patin $^{20}$ s'applique bien à Renaudot dont la dégringolade fut rapide.

Le $1^{\text {er }}$ mars 1644, La Grand'Chambre confirme la sentence du Châtelet le condamnant pour exercice illégal de la médecine : il a tout perdu hormis la Gazette et le Bureau d'adresse.

On attribue à Guy Patin un exécrable torchon de huit pages qui parut à Paris en 1644 : Le Nez pourri de Théophraste Renaudot, grand gazetier de France et espion de Mazarin, appelé dans les Chroniques Nebulo hebdomarius, de patria Diabolorum. Avec sa vie infâme et bouquine, récompensée d'une vérole euripienne, ses usures, la décadence de ses Monts-de-piété, et la ruine de tous ses fourneaux et alambics (excepté celle de sa conférence, rétablie depuis quinze jours) par la perte de son procès contre les docteurs de la Faculté de médecine de Paris ${ }^{21}$. Il se termine par ce rondeau «Sur le nez pourri de Théophraste Renaudot, alchimiste, charlatan, empirique, usurier comme un Juif, perfide comme un Turc, méchant comme un renégat, grand fourbe, grand usurier, grand gazetier de France » :

Un pied de nez servirait davantage

À ce fripier, docteur du bas étage

Pour fleurer [flairer] tout, du matin jusqu'au soir ;

Et toutefois on dirait à le voir,

19 « Plura dicam: animum ipsum ex Nasi purulentia infici uolunt Physiognomones, unde illis est axioma; Corruptum Nasum sequitur corruptio morum, quod est probabile: ex isto enim Nasorum genere, qui rancidulo ore loquuntur, hoc est $\alpha \eta \delta \omega \varsigma \kappa \alpha \iota ~ \delta v \sigma \chi \varepsilon \rho \omega \varsigma$, uulgo nebulones sunt, Ridiculi, Effrænes, Nefarii, Ardeliones, Vafri, Dolosi, Obscæni, Turbulenti, mendaces, maligni, inuidi, quadruplatores, flagitiosi, infames, contumeliosi, facinorosi, nulla uirtute redempti, a uitiis ægri solaque libidine fortes, industria ficti, et a Natura facti ad fraudem et calumniam ». Trad. L.C. - Les huit adjectifs latins que j'ai transcrits avec une majuscule initiale forment l'acrosctiche RENAVDOT, mais ma version française a dû tricher un peu (sur Vafri, «fourbes ", que j'ai traduit par « Vauriens ») pour reproduire cette acrobatie.

${ }^{20}$ Lettre à Charles Spon datée du 29 avril 1644, parlant de la disgrâce de $\mathrm{M}^{\text {me }}$ de Hautefort, favorite du feu roi Louis XIII.

${ }^{21}$ Dans ce titre : Nebulo hebdomarius, de patria Diabolorum signifie « Vaurien semainier, venu de la patrie des Diables [Loudun] », bouquine, débauchée, et euripienne, invétérée. 
Que c'est un dieu de la chinoise plage.*

Mais qu'ai-je dit ? c'est plutôt un fromage

Où sans respect, la mite a fait ravage ;

Pour le sentir, il ne faut point avoir

Un pied de nez.**

Le fin camus touché de ce langage,

Met aussitôt un remède en usage,

Où d'Esculape il ressent le pouvoir :

Car s'y frottant, il s'est vu recevoir

En plein Sénat, tout le long du visage,

Un pied de nez.***

* «Les dieux de la Chine ont le nez écaché [écrasé et aplati]» (note de l'éditeur du livre). Plage est à prendre pour rivage, ou pour désert.

** Un nez long d'un pied (32,4 centimètres).

*** « On dit qu'un homme a eu un pied de nez, quand il a été trompé dans ses espérances » (Furetière).

Le 17 mai 1644, un arrêt du Parlement officialise les consultations charitables de la Faculté de médecine créées en 1639.

Le 21 octobre suivant, Siméon Courtaud, doyen de Montpellier, prononce un violent discours contre les Parisiens : en défendant Renaudot, il étend la querelle à l'arrogance de la Faculté et à ses pratique rétrogrades qui excluent les remèdes chimiques.

En décembre 1648, sous la pression du parti antimonial qui monte en puissance, la Faculté de médecine confère le doctorat à Isaac et Eusèbe Renaudot.

\section{Suites et fin}

Renaudot meurt, « gueux comme un peintre $»^{22}$, le 25 octobre 1653.

Ridiculisé par ses chimères médicales et ruiné pas ses mécomptes familiaux, Patin décède le 30 mars 1672. Publiées pour la première fois en $1693^{23}$, ses lettres françaises sont jugées scandaleuses mais remportent un franc succès.

En 1849, Jean-Henri Reveillé-Parise en donne une édition extensive ${ }^{24}$ et provoque un engouement renouvelé pour Patin. Ému par la virulence et l'injustice des attaques qu'on y lit contre Renaudot, Georges Gilles de La Tourette écrit en 1884 un livre pour louer le créateur du journalisme en France et le martyr de la charité publique ${ }^{25}$. En 1893, grâce à une souscription publique, une statue de Renaudot est inaugurée

\footnotetext{
${ }^{22}$ Lettre à Claude Belin, le 12 novembre 1653.

${ }^{23}$ Voir supra, note 16.

${ }^{24}$ Lettres de Gui Patin, Paris, J.B. Baillière, 1846, 3 vol., contenant 836 lettres.

${ }^{25}$ Voir supra, note 3.
} 


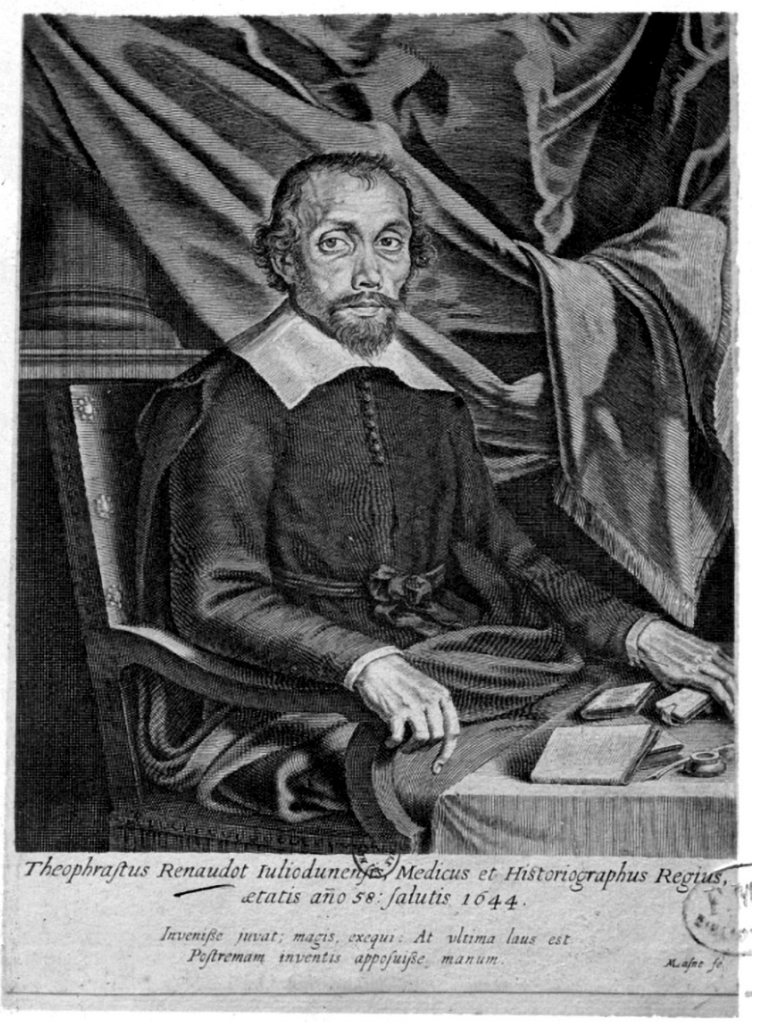

Figure 1. Portrait de Théophraste Renaudot, «natif de Loudun, médecin et historiographe du roi, en 1644, à l'âge de 58 ans », avec sa devise (traduite du latin) : « Il est utile d'avoir fait des découvertes ; plus utile encore de les avoir développées ; mais l'ultime gloire vient d'y avoir mis la dernière main » (Banque d'images et de portraits de la BIU-Santé, CIPB0301, libre de droits).

sur l'île de la Cité, là où se situait le Bureau d'adresse, face au Palais de justice ; le bronze en est fondu en 1941.

\section{En 1926, est fondé le Prix Théophraste Renaudot :}

Pour occuper les loisirs que l'Académie Goncourt laisse chaque année aux informateurs littéraires pendant qu'elle délibère pour l'attribution de son prix. Ne se proposant en aucune façon de concurrencer les jurys depuis longtemps consacrés, dix informateurs appartenant à dix grands journaux parisiens ont décidé de placer leur prix sous l'invocation de l'illustre père du journalisme en France ${ }^{26}$.

${ }^{26}$ La Vie de Théophraste Renaudot par **********, Paris, Gallimard, NRF, 1929. 


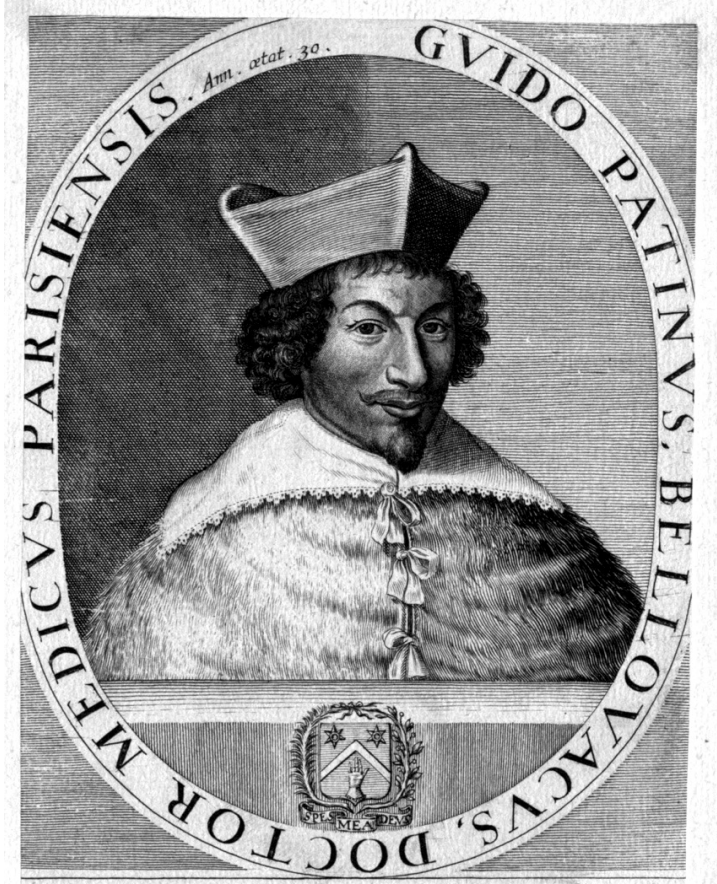

Galeni vindex, peregrini dogmatis ofor

Errorúmque, ifta cesnitur effigie.

I.D.N.T.B.B.

Figure 2. Portrait de Guy Patin, «natif du Beauvaisis, docteur en médecine de Paris, à l'âge de 30 ans » (1630-1631), avec sa louange (écrite par son ami Jean de Nully, ici traduite du latin) : « Ce portrait dépeint le défenseur de Galien, le pourfendeur du dogme étranger et des erreurs »

(Österreichische Nationalbibliothek, Bildarchiv und Grafiksammlung, libre de droits).

\section{6. Épilogue}

Nos deux ennemis méritent une place dans les anthologies du XVII ${ }^{\mathrm{e}}$ siècle. Disserterait-on pourtant encore sur leur duel s'ils s'étaient seulement méprisés ? Sûrement non, car mépriser vraiment, c'est dédaigner et ne pas répondre : voilà pourquoi Furetière disait : « il n'y a point de dette sitôt payée que le mépris $»^{27}$. Le mépris n'est pas littéraire parce qu'il est muet. Ce paradoxe ne m'a pas empêché d'évoquer ici la haine tenace que se vouaient deux médecins qui avaient choisi des moyens radicalement opposés pour parvenir à accomplir leur idéal. Chacun a sa

${ }^{27}$ A. Furetière, op. cit. 
statue dans son pays natal : on a inauguré celle de Renaudot à Loudun (Haut-Poitou) en 1894, et celle de Patin à Hodenc-en-Bray (Picardie) en 1898. Le plus sage aujourd'hui est de les renvoyer dos à dos, en laissant à leurs bronzes le loisir de se mépriser vraiment, très loin l'un de l'autre.

\section{Bibliographie}

Boisse, Jacques, Description d'un médicament appelé polychreston, dispensé publiquement par Jacques Boisse, maître apothicaire en la ville de Loudun, le 4 décembre 1619. Avec la harangue faite sur ce sujet par Théophraste Renaudot, docteur en médecine, conseiller et médecin du roi, devant Messieurs les députés des Églises réformées de ce royaume, assemblés à Loudun par permission de sa Majesté. Dédié à Messieurs de l'Assemblée (Loudun, Quentin Maréchal, 1619, in-4º). Mêlant 83 extraits d'origine végétale ou animale, ce polychreston était un plagiat manifeste du polychreste que Jean Pidoux, médecin de Poitiers, avait décrit dans son livre intitulé Pestis cura et polychresti descriptio [Guérison de la peste et description du polychreste] (Poitiers, Jean Blancet, 1605, in- $8^{\circ}$ ).

Capron,Loïc, Correspondance complète et autresécrits de GuyPatin, http://www.biusante.parisdescartes.fr/ patin/, consulté le 09.01.2019

G. Gilles de la Tourette, Théophraste Renaudot d'après des documents inédits, Paris, Plon, 1884

Commentaires de la Faculté de médecine de Paris, tome XIII (1636-1652), fo 141 vo142 ro Medic@, http://www.biusante.parisdescartes.fr/histmed/medica/page?ms00001_00024x13\&p=292, consulté le 16.01.2019

Danielis Sennerti D. Medici Opera omnia in tres tomos distincta [Euvres complètes de Daniel Sennert, docteur en médecine, réparties en trois tomes] (Paris, par une Société de libraires, 1641, in- $\mathrm{f}^{\mathrm{0}}$ )

Furetière, Antoine (1619-1688), Dictionnaire universel, La Haye, A. et R. Leers, 1690, fo 3Qqq $2 \mathrm{r}^{\circ}$

Commentaires de la Faculté de médecine de Paris, tome XIII (1636-1652), fo 48 ro , Medic@, http://www. biusante.parisdescartes.fr/histmed/medica/page?ms00001_00024x13\&p=97, consulté le 16.01.2019

Lettres patentes de Louis XIII imprimées à la fin des Consultations charitables pour les malades. Dédiées à Monseigneur de Noyers, secrétaire d'État (Paris, Bureau d'Adresse, 1640, in-4º)

Lettres choisies de Feu Monsieur Guy Patin, docteur en médecine de la Faculté de Paris et professeur au Collège royal. Dans lesquelles sont contenues plusieurs particularités historiques, sur la vie et la mort des savants de ce siècle, sur leurs écrits et sur plusieurs autres choses curieuses, depuis l'an 1645 jusqu'en 1672 (Francfort, pour J.L. Du-Four, 1683, in-12², pour la première des nombreuses éditions parues aux $\mathrm{XVII}^{\mathrm{e}}$ et $\mathrm{XVIII}^{\mathrm{e}}$ siècles)

Lettres de Gui Patin, Paris, J.B. Baillière, 1846, 3 vol., contenant 836 lettres

La vie de Théophraste Renaudot par **********, Paris, Gallimard, NRF, 1929

Réponse à l'Examen de la requête présentée à la reine par $M^{\mathrm{e}}$ Théophraste Renaudot, portée à son auteur par Machurat, compagnon imprimeur (Paris, sans nom, 1644, in- $4^{\circ}$ ). Renaudot y répondait anonymement à l'Examen de la Requête présentée à la reine par le gazetier (sans lieu ni nom, 4 novembre 1643 , in- $4^{\circ}$ )

Loïc Capron, né le 6 décembre 1949 à Arras (France, Pas-de-Calais), est médecin. Retraité depuis septembre 2019, il a été professeur de l'Université Paris-Descartes et praticien hospitalier en médecine interne à l'Assistance publique-hôpitaux de Paris ; il a principalement orienté sa pratique et ses recherches sur les maladies vasculaires et sur la sémiologie clinique. Depuis la fin de 2000, il travaille à une édition électronique intégrale des œuvres de Guy Patin : transcription, traduction française du latin (chaque fois que nécessaire), annotation et indexation. Librement accessible sur la Toile 
(http://www.biusante.parisdescartes.fr/patin/), ce site a été régulièrement corrigé et augmenté depuis la mise en ligne de la Correspondance française en mars 2015 : Correspondance latine en juin 2018, nombreux autres écrits en février 2019 et les cinq ana qu'on lui attribue en avril 2021. Ce très vaste corpus est une source intarissable de renseignements concernant principalement la période centrée sur 1550-1670, tant en médecine que dans les autres disciplines (religions, philosophie, arts et lettres, politique). Une des grandes richesses de cette édition est son interactivité qui permet de fructueux échanges entre l'auteur et les lecteurs, très souvent suivis de corrections.

\begin{tabular}{|c|c|}
\hline cc)creative & $\begin{array}{l}\text { (C) by the author, licensee Łódź University - Łódź University Press, } \\
\text { Łódź, Poland. This article is an open access article distributed under } \\
\text { the terms and conditions of the Creative Commons Attribution license } \\
\text { CC-BY-NC-ND } 4.0 \text { (https://creativecommons.org/licenses/by-nc-nd/4.0/) }\end{array}$ \\
\hline & Received: 2019-01-25; Accepted: 2020-10-05 \\
\hline
\end{tabular}

